\title{
Ethnologies
}

Christopher A. Scales. Recording Culture. Powwow Music and the Aboriginal Recording Industry on the Northern Plains. (Durham et Londres: 2012, Duke University Press. Pp.368. ISBN : 978-0-8223-5338-6)

\section{Ons Barnat}

Volume 35, numéro 1, 2013

URI : https://id.erudit.org/iderudit/1026459ar

DOI : https://doi.org/10.7202/1026459ar

Aller au sommaire du numéro

Éditeur(s)

Association Canadienne d'Ethnologie et de Folklore

ISSN

1481-5974 (imprimé)

1708-0401 (numérique)

Découvrir la revue

Citer ce compte rendu

Barnat, O. (2013). Compte rendu de [Christopher A. Scales. Recording Culture. Powwow Music and the Aboriginal Recording Industry on the Northern Plains. (Durham et Londres: 2012, Duke University Press. Pp.368. ISBN :

978-0-8223-5338-6)]. Ethnologies, 35(1), 178-185.

https://doi.org/10.7202/1026459ar d'utilisation que vous pouvez consulter en ligne. 
Christopher A. Scales. Recording Culture. Powwow Music and the Aboriginal Recording Industry on the Northern Plains. (Durham et Londres: 2012, Duke University Press. Pp.368. ISBN : 978-0-8223-5338-6)

Recording Culture. Powwow Music and the Aboriginal Recording

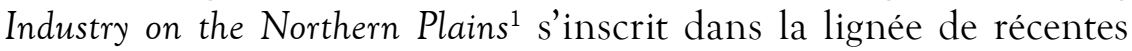
contributions d'ethnomusicologues à l'étude du phénomène de l'enregistrement en studio, à des fins commerciales, de genres musicaux "traditionnels" par des acteurs locaux. Depuis les recherches pionnières de Jocelyne Guilbault sur le zouk dans les années 1990, et les travaux de Paul Théberge sur les rapports entre musique et technologie $(1997,2004)$, plusieurs auteurs ont fait du studio d'enregistrement leur principal terrain de recherche : Louise Meintjes dans des studios de Johannesburg, Beverly Diamond au Canada, Karl Neuenfeldt en Australie, Thomas Porcello aux ÉtatsUnis, Frederick Moehn au Brésil, Eliot Bates en Turquie, mais aussi Peter Greene au Népal, et moi-même en Amérique centrale. Les enjeux méthodologiques et épistémologiques liés à l'analyse de cette «boîte noire " de l'ethnomusicologie n'ont certainement pas fini de grossir cette liste - non exhaustive - de recherches ayant pris pour terrain cet espace dynamique de création, investi par des acteurs divers réunis dans le but précis d'y enregistrer de la musique...

Ce premier ouvrage de l'ethnomusicologue états-unien Christopher A. Scales, aujourd'hui professeur associé à la Michigan State University, trouve son origine dans la thèse qu'il a soutenue en 2004 sous la direction de Bruno Nettl. À la fois musicien, chercheur, ingénieur du son et réalisateur (il compte à son actif plusieurs disques, parus chez Arbor Records et War Pony Records), Scales livre ici brillamment les fruits de ses recherches sur la musique powwow nord-américaine, amorcées il y a près de vingt ans. En se basant sur des données extraites aussi bien au cour du studio d'enregistrement qu'à l'occasion de rassemblements powwows, l'auteur nous plonge dans l'univers méconnu des musiciens autochtones des Prairies, les suivants pas à pas des powwow grounds aux sessions d'enregistrement en studio.

1. À ne pas confondre avec l'ouvrage Recording Culture. Audio Documentary and the Ethnographic Experience, paru en 2009 chez Sage. 
Avec un tel terrain double (à l'intérieur et à l'extérieur du studio), ce livre fascinera aussi bien les amateurs de musiques autochtones nord-américaines que les chercheurs s'intéressant au rôle de la technologie dans la création et la préservation de musiques dites «traditionnelles ». À partir du cas de l'enregistrement de la musique powwow, Scales décrypte avec lucidité une série de dynamiques sociales animant l'ensemble du " monde powwow » (aux niveaux macro et micro), tout en mettant le doigt sur un point clé de la recherche ethnomusicologique depuis les débuts de cette discipline : la rencontre motivée entre des "autochtones musiciens et des enregistreurs non autochtones "(2). Définissant son livre comme " une étude ethnographique de la culture powwow contemporaine ", l'ethnomusicologue se lance pour défi d'analyser le lien dynamique entre la pratique de la musique powwow en contexte cérémoniel et son enregistrement en studio. Pour ce faire, il choisit de structurer son ouvrage en deux grandes sections, qui peinent à s'harmoniser. En effet, si la première partie (composée de trois chapitres) se consacre surtout à la description minutieuse de la musique et de la culture pow-wow en contexte "traditionnel ", la deuxième partie nous fait progressivement pénétrer dans le monde du studio d'enregistrement, après avoir été familiarisé aux rouages de l'industrie discographique autochtone nord-américaine.

Passer ainsi d'une lourde monographie (somme toute très classique depuis les débuts de l'ethnomusicologie) à une ethnographie pointue du studio (qui inclut des considérations portant sur des paramètres technologiques complexes) ne semble pas trop déranger Scales, qui se justifie en avançant qu'il a cherché « à analyser la production et la consommation des enregistrements de musique powwow comme une sorte de boucle fermée, où chaque contexte influence l'autre et devient de plus en plus interdépendant » (243). Cependant, force est de constater que cette division structurelle ne fait que reproduire une dichotomisation entre " tradition " et " modernité ", termes que l'auteur ne manque pourtant pas de critiquer longuement dans son dernier chapitre. Pour lui, la relation entre les enregistrements powwows en studio et la pratique «traditionnelle » de cette musique serait bien plus complexe : « la force culturellement significative de la tradition est créée au sein, et est d'ailleurs entièrement dépendante, des conditions structurelles de la modernité " (261). 
Cet ouvrage - qui se veut une première recherche sur la musique powwow en studio, faisant suite à de nombreuses études portant sur le powwow en tant qu'acte performantiel en contexte «traditionnel »prend pour point de départ un questionnement concernant les raisons qui motiveraient les acteurs des powwows à vouloir enregistrer leur musique. En analysant les différences entre des pièces jouées lors de cérémonies et d'autres enregistrées en studio, l'auteur va chercher à comprendre quels seraient les principes esthétiques qui gouvernent la musique powwow nord-américaine. Au niveau social, ce sont plutôt les liens entre compétitions de powwow (qui pullulent en été sur les Prairies) et les enregistrements discographiques de musique powwow (qui se vendent et se trouvent surtout dans ces mêmes compétitions) qui serviront de fil conducteur à l'ensemble du livre. Tout cela dans le but de décrypter le « réseau complexe d'interactions sociales et d'allégeances esthétiques générées par la participation autochtone dans le circuit powwow des Prairies » (4).

La première partie, intitulée «La culture powwow des Plaines du nord ", comporte trois chapitres, abordant tour à tour des considérations sur la pratique de la musique powwow, sur l'esthétique de cette musique, et enfin sur les acteurs qui la font vivre. Malgré quelques longueurs (dues à la densité des informations fournies), ces trois premiers chapitres offrent une claire définition de la cérémonie powwow et de la musique qui l'accompagne. On rentre ainsi progressivement dans le monde peu médiatisé des musiciens autochtones nord-américains, avec tout d'abord une description ethnographique précise d'un powwow de compétition, où différents groupes de tambours (drum groups) s'affrontent pour gagner des prix pouvant aller jusqu'à 20000 \$. Après la "grande entrée ", sorte de parade où tous les acteurs impliqués (musiciens, chanteurs, danseurs...) défilent selon des normes préétablies, des juges s'affairent durant trois jours (généralement en fin de semaine) autour des groupes de tambours afin de désigner les lauréats de différentes catégories (regroupées par tranches d'âge). Ce système de récompenses pécuniaires est au contraire absent des powwows " traditionnels", détaillés dans la deuxième partie de ce premier chapitre. Présentés comme d'importance moindre (en terme d'achalandage et de retombées financières), les powwows « traditionnels » demeurent prisés par l'ensemble des groupes de tambours pour qui la participation bénévole à ces cérémonies représente une façon de "rester modeste ", 
de se "sentir bien autour du tambour ", de " rendre les gens heureux »... sans entrer dans une logique capitaliste de compétition : "Dans les powwows "traditionnels" on chante pour le "plaisir" et non pas pour l'argent qui peut être gagné à travers la compétition » (59).

Après ce premier chapitre de contextualisation, Scales nous propose de rentrer plus en détails dans les "chansons powwows " que l'on entend dans les powwows de compétition. Se plaçant du côté des juges chargés d'évaluer les performances des groupes de tambours inscrits aux compétitions, on découvre une série de critères esthétiques définissant ce qui constituerait une "bonne " performance. Un premier tableau, page 86, nous renseigne sur les six différentes catégories de danses structurant le déroulement d'un powwow de compétition, catégories elles-mêmes regroupées en quatre classes d'âge. D'autres critères esthétiques sont ensuite mis en évidence - cette fois-ci à partir du point de vue des musiciens - après l'analyse d'extraits d'entretiens extrêmement vivants, qui témoignent de la dynamique des échanges entre le chercheur et les musiciens interviewés.

Le troisième chapitre, "Groupes de tambour et chanteurs ", se consacre à « détailler les arrangements sociaux qui se forgent pendant un powwow " (113), à partir des cas de deux groupes de tambour que l'auteur a côtoyé pendant les étés 1999 et 2000 : les Spirit Sand Singers et les Northern Wind Singers. En suivant leurs pas sur le «sentier du powwow ", on comprend l'importance de la "culture du voyage " pour ces musiciens, pris dans une logique financière dans laquelle l'accès à des prix lors de compétitions conditionne le remboursement de leurs frais de voyages. Ce chapitre s'achève sur des précisions sur l'organisation sociale d'un groupe de tambour, avant de faire un bref lien entre la participation à des compétitions et l'apparition de labels et de studios dédiés à l'enregistrement de musique powwow.

La deuxième partie du livre s'ouvre sur un historique de l'industrie de l'enregistrement discographique de la musique powwow dans l'Ouest canadien. Scales pose ensuite deux objectifs : d'une part, "décrire et caractériser la structure institutionnelle et économique des labels de musique powwow " (145), et de l'autre, de comprendre les mécanismes des interactions au sein d'Arbor Records, un label indépendant manitobain spécialisé dans les musiques autochtones. 
Pour l'auteur, la naissance de ces labels coïncide avec l'apparition des grands powwows de compétition, subventionnés par des casinos implantés dans des réserves amérindiennes. En effet, l'entrée d'argent générée par ces institutions se trouve répercutée sur toute l'économie de la communauté powwow, les groupes de tambours désireux de remporter des prix étant tenus d'avoir réalisé des enregistrements discographiques commercialisés, garants pour leur public de leur "professionnalisme » et de leur « réputation ». Empruntant un marché très restreint, la commercialisation de musique powwow passe par un circuit de distribution spécialisé et en marge des musiques mainstream nord-américaines, notamment à travers le réseau des vendeurs ambulants qui se déplacent de powwow en powwow, ainsi que dans les petits magasins indépendants (appelés one-stops ou mom-and-pop stores) qui jonchent les Prairies canadiennes.

C'est finalement dans le cinquième chapitre de ce livre que sont abordées de front les problématiques liées à l'enregistrement en studio de la musique powwow "traditionnelle ». L'accent y est mis sur le processus créatif en studio, lieu de " médiation " et de négociation entre des personnes aux intérêts esthétiques et commerciaux différents. Scales base tout son argumentaire sur l'observation ethnographique de deux enregistrements de musique autochtone, réalisés par Arbor Records et auxquels l'auteur a largement participé en tant qu'ingénieur du son. Cette proximité du chercheur avec son objet d'étude donne ici lieu à une description précise et vivante du processus de création discographique en studio, avec une série d'anecdotes croustillantes sur des sessions d'enregistrements, définies comme « hautement interactives " (202) entre les musiciens, ingénieurs du son et réalisateurs impliqués.

Dans le chapitre suivant, également centré sur ce qui se passe en studio, on rentre encore plus en détails dans l'univers de l'enregistrement discographique de la musique powwow. À partir de ses observations in situ, Christopher Scales fait ressortir l'importance du concept de liveness aussi bien pour les musiciens autochtones que pour les employés non autochtones d'Arbor Records. En effet, une des caractéristiques fondamentales du "son » d'un enregistrement powwow résiderait dans sa capacité à faire entendre et ressentir les aspects propres aux performances en direct et en contexte cérémoniel. Cela va sans dire que la transplantation de la musique powwow de 
la réserve au studio ne se réalise pas sans difficultés, les sollicitations esthétiques des musiciens ne coïncidant pas forcément avec les impératifs techniques qui conditionnent le travail des ingénieurs du son et du réalisateur.

Le septième et dernier chapitre avant la «Coda » reprend la question qui avait intrigué Scales au début de sa recherche : pourquoi les musiciens powwow veulent-ils s'enregistrer? Opérant un retour sur l'ensemble de son livre, l'ethnomusicologue dégage ainsi trois moteurs de cette dynamique : l'argent (même si les gains occasionnés par la vente de disques servent surtout à couvrir les frais de subsistance des groupes de tambour), la réputation et le prestige (le fait d'avoir sur le marché plusieurs disques représente pour la communauté powwow un gage de qualité et de professionnalisme) et la préservation de la culture et de l'histoire (une chanson enregistrée devenant une partie intégrante du patrimoine culturel powwow).

Avant de conclure son livre, Christopher Scales se lance dans un vibrant plaidoyer (262-267) pour une ethnomusicologie de l'enregistrement en studio, qui viserait à balayer les velléités ethnocentristes des " enregistrements ethniques académiques " (d'après les termes de Kay Shelemay). Suite à son expérience double en tant qu'ethnomusicologue et ingénieur du son engagé par une maison de disques indépendante, Scales soutient en effet que l'exercice actuel de l'ethnomusicologie peut (et devrait) se faire à travers la réalisation d'enregistrements commerciaux qui « challengent le discours du réalisme documentaire caractéristique [des disques ethnico-académiques] : un discours qui relie la performance en direct à l'authenticité " (266). Pour lui, il est important de dépasser ce paradigme épistémologique sévèrement ancré dans la discipline, en reconnaissant que la pratique $\mathrm{du}$ " réalisme documentaire [...] n'est qu'un choix parmi d'autres, choix qui est historiquement et culturellement déterminé »(267).

Au final, l'ouvrage Recording Culture. Powwow Music and the Aboriginal Recording Industry on the Northern Plains pointe du doigt une série de propositions épistémologiques à même d'opérer un changement de paradigme pour l'ensemble de la discipline ethnomusicologique. Tout d'abord, en passant de l'analyse du matériau musical (l'œuvre, le disque, le concert...) à celle du processus 
créateur en studio, on arriverait à mettre en évidence les dynamiques sociales à l'origine de tout acte créateur : " comprendre le processus de l'enregistrement en studio en termes musicologiques permet de cerner la spécificité des relations sociales au sein d'un contexte plus large » (203). En ce qui concerne le rôle et la place du studio d'enregistrement dans la recherche ethnomusicologique, Scales affirme que le studio « existe à l'intersection de la création musicale et de la commercialisation ", étant " une institution commerciale visant à créer des produits esthétiques ». De plus, l'auteur soutient que la fabrication de l'esthétique des enregistrements de musique powwow, basée sur la notion de liveness, "se trouve à l'intersection entre la technologie et la "tradition", un "entre-deux" [...] entre les terres du powwow et le studio d'enregistrement " (255). L'importance centrale des enregistrements résiderait donc dans leurs facultés de « synthétiser une série de valeurs esthétiques et de positions sociales "(256).

Malgré une première partie assez lourde (offrant tout de même un panorama détaillé de la musique powwow avant son entrée en studio), l'emploi de données un peu dépassées (datant de dix à quinze ans) et le manque notoire de liens vers des pages internet (et surtout vers des vidéos, absentes du disque compact), ce premier livre de Christopher A. Scales représente une excellente contribution aux études ethnomusicologiques focalisées sur l'analyse du rôle de la technologie du studio d'enregistrement dans la création, la transformation, la préservation et la diffusion de genres musicaux " traditionnels ». Clairement passionné par sa recherche, l'ethnomusicologue-ingénieur du son-réalisateur expose avec grand discernement les défis qui lui ont été posés durant la tenue de sa recherche doctorale, défis qu'il a su surmonter avec brio. Plus encore, les questionnements épistémologiques auxquels il a été confronté sauront trouver écho auprès des nouvelles générations de chercheurs qui expérimentent sur leurs terrains des situations identiques, n'étant désormais plus les seuls maîtres à bord de la technologie - comme cela a toujours été le cas jusqu'à très récemment.

La miniaturisation et la diffusion mondiale des appareils technologiques (aussi bien dans l'enregistrement sonore qu'audiovisuel ou photographique), couplées à une augmentation frénétique des échanges et des communications (via l'omnipotent internet), ont aujourd'hui pour effet de décupler les possibilités créatrices de 
l'homme contemporain. Dans un monde où la technologie semble ainsi changer les vies de plus en plus d'humains, il y a de quoi penser que l'ethnomusicologie - bien que née en révolte à la suprématie ethnocentrique de la musicologie historique européenne - n'a pas fini de se développer, pour tenter de répondre aux questions que nous pose la musique, ce « suprême mystère des sciences de l'homme, celui contre lequel elles butent et qui garde la clé de leur progrès " comme le devisait si savamment Claude Lévi-Strauss (1964 : 26), il y a près de cinquante ans dans Le cru et le cuit.

Ons Barnat Université de Montréal

\section{Références}

Lévi-Strauss, Claude, 1964, Le cru et le cuit, Paris, Plon.

Gérard Bouchard. L'Interculturalisme. Un point de vue québécois. (Montréal, Les Éditions du Boréal, 2012, Pp. 288. : ISBN 978-2-7646-2207-0)

Cet ouvrage du sociologue et historien Gérard Bouchard, propose un modèle d'interculturalisme comme paradigme pour la gestion de la diversité ethnoculturelle du Québec. La publication s'inscrit dans le sillon de la "Commission de la consultation sur les pratiques d'accommodement reliées aux différences culturelles » (mieux connue sous le nom de Commission Bouchard-Taylor) qui s'est déroulée dans tout le Québec en 2007 et 2008, et fut coprésidée par le philosophe Charles Taylor et par Gérard Bouchard lui-même. Cette publication est produite dans un contexte où, tant en Amérique du Nord qu'en Europe, les gouvernements sont à la recherche de nouveaux outils intellectuels pour gérer la diversité sociale et culturelle. Cela s'explique principalement par l'inefficacité des modèles précédents, à résoudre la 